\title{
A construction of closed helices with self-intersections in a complex projective space by using submanifold theory
}

\author{
Toshiaki ADACHI* and Sadahiro MAEDA
}

(Received January 29, 1998)

\begin{abstract}
In this paper we construct a certain class of closed helices with self-intersections in a complex projective plane. This is a nice class of curves which are not generated by global Killing vector fields.

Key words: helix, self-intersection, complex projective space, isometric imbedding, curvature, complex torsion.
\end{abstract}

\section{Introduction}

The main purpose of this paper is to give a certain class of closed helices with self-intersections in a complex projective space by using a well-known isometric imbedding. In a real space form, which is a Euclidean space $\mathbb{R}^{n}$, a standard sphere $S^{n}$, or a hyperbolic space $H^{n}$, it is well-known that a smooth curve is a helix if and only if it is generated by a Killing vector field on this space. In a symmetric space of rank one, every circle, that is a helix of order 2 , is generated by a Killing vector field. From this point of view we are interested in the difference between the set of helices of order $d(\geq 3)$ and the set of curves generated by Killing vector fields in a symmetric space of rank one. Needless to say, in any Riemannian manifold $M$ every integral curve of a Killing vector field is a helix. But in general, a helix is not necessarily an integral curve of some Killing vector field in $M$.

One of the most important properties of integral curves of Killing vector fields is that they do not have any self-intersection points. In this paper we pay attention to self-intersection points of helices in a complex projective space. In section 2 we give a class of helices with self-intersection points by using an isometric imbedding of a flat torus into a complex projective plane defined in [4]. This imbedding maps geodesics in a torus to circles

1991 Mathematics Subject Classification : 53C22, 53C42.

${ }^{*}$ This research was partially supported by Grand-in-Aid for Encouragement of Young Scientists (No. 09740055), Ministry of Education, Science and Culture 
in a complex projective plane. We here study the image of circles by this imbedding. In section 3 we also study helices which are generated by Killing vector fields by using the Veronese imbedding of degree 2 .

\section{Helices on a complex space form}

We shall start with reviewing the Frenet formula for a smooth Frenet curve in a Riemannian manifold $M$ with Riemannian metric $\langle$,$\rangle (cf. [3]).$ A smooth curve $\gamma=\gamma(s)$ parametrized by its arclength $s$ is called a Frenet curve of proper order $d$ if there exist orthonormal frame fields $\left\{V_{1}=\right.$ $\left.\dot{\gamma}, \ldots, V_{d}\right\}$ along $\gamma$ and positive functions $\kappa_{1}(s), \ldots, \kappa_{d-1}(s)$ which satisfy the following system of ordinary equations

$$
\nabla_{\dot{\gamma}} V_{j}(s)=-\kappa_{j-1}(s) V_{j-1}(s)+\kappa_{j}(s) V_{j+1}(s), \quad j=1, \ldots, d,
$$

where $V_{0} \equiv V_{d+1} \equiv 0$ and $\nabla_{\dot{\gamma}}$ denotes the covariant differentiation along $\gamma$. We call Equation (1.1) the Frenet formula for the Frenet curve $\gamma$. The functions $\kappa_{j}(s)(j=1, \ldots, d-1)$ and the orthonormal frame $\left\{V_{1}, \ldots, V_{d}\right\}$ are called the curvatures and the Frenet frame of $\gamma$, respectively. Here we note that we do not allow the curvature function to be vanished at some point. Therefore curves with inflection points, such as $y=x^{3}$ on a Euclidean $x y$-plane, are not Frenet curves. The definition of Frenet curves so seems a bit artificial. This is because we need the Frenet frame of a Frenet curve to be continuous and we suppose curvature functions to be positive. Once we allow curvature functions to have variable signature, we can treat more general curves. But for the sake of simplicity and to follow classical treatment of smooth curves, we treat Frenet curves. So the reader should not stick to this point.

A Frenet curve is called a Frenet curve of order $d$ if it is a Frenet curve of proper order $r(\leq d)$. For a Frenet curve of order $d$ which is of proper order $r(\leq d)$, we use the convention in (1.1) that $\kappa_{j} \equiv 0(r \leq j \leq d-1)$ and $V_{j} \equiv 0(r+1 \leq j \leq d)$. In real space forms, it is known that a smooth curve is generated by some Killing vector field if and only if all its curvatures are constant. We call a smooth curve a helix when all its curvatures are constant. A helix of order 1 is nothing but a geodesic. A helix of order 2 , that is a curve which satisfies $\nabla_{\dot{\gamma}} V_{1}(s)=k V_{2}(s), \nabla_{\dot{\gamma}} V_{2}(s)=-k V_{1}(s)$, $V_{1}(s)=\dot{\gamma}(s)$, is called a circle of curvature $k$.

We now restrict ourselves to Frenet curves on Kähler manifolds. Let 
$M$ be an $n$-dimensional Kähler manifold with complex structure $J$ and Riemannian metric $\langle$,$\rangle . For a Frenet curve \gamma=\gamma(s)$ in $M$ of order $d(\leq 2 n)$ with the associated Frenet frame $\left\{V_{1}, \ldots, V_{d}\right\}$, we set $\tau_{i j}(s)=$ $\left\langle V_{i}(s), J V_{j}(s)\right\rangle$ for $1 \leq i<j \leq d$ and call them its complex torsions. In the study of Frenet curves in a Kähler manifold their complex torsions play an important role. We have the following fundamental result on curves in complex space forms, that is complete simply connected Kähler manifolds of constant holomorphic sectional curvature, which are complex Euclidean space $\mathbb{C}^{n}$, complex projective space $\mathbb{C} P^{n}$, and complex hyperbolic space $\mathbb{C} H^{n}$.

Fact ([3]) In a complex space form $M$, a Frenet curve is generated by a holomorphic Killing vector field on $M$ if and only if all its curvatures and all its complex torsions are constant functions.

In a complex space form, every circle is generated by some Killing vector field. But of course there are many helices which are not generated by Killing vector fields on a complex space form. For example, let $\gamma=\gamma(s)$ be a helix of proper order 3 on a Kähler manifold. The complex torsions of $\gamma$ then satisfy the following equations:

$$
\left\{\begin{array}{l}
\tau_{12}^{\prime}=\kappa_{2} \tau_{13}, \\
\tau_{13}^{\prime}=-\kappa_{2} \tau_{12}+\kappa_{1} \tau_{23}, \\
\tau_{23}^{\prime}=-\kappa_{1} \tau_{13},
\end{array}\right.
$$

where $\kappa_{1}, \kappa_{2}$ denote the curvatures of the helix. By solving them, we have

$$
\left\{\begin{array}{l}
\tau_{12}(s)=\alpha_{1} \sin \sqrt{\kappa_{1}^{2}+\kappa_{2}^{2}} s+\alpha_{2} \cos \sqrt{\kappa_{1}^{2}+\kappa_{2}^{2}} s+\alpha_{3} \\
\tau_{13}(s)=\frac{\sqrt{\kappa_{1}^{2}+\kappa_{2}^{2}}}{\kappa_{2}}\left(\alpha_{1} \cos \sqrt{\kappa_{1}^{2}+\kappa_{2}^{2}} s-\alpha_{2} \sin \sqrt{\kappa_{1}^{2}+\kappa_{2}^{2}} s\right) \\
\tau_{23}(s)=-\frac{\kappa_{1}}{\kappa_{2}}\left(\alpha_{1} \sin \sqrt{\kappa_{1}^{2}+\kappa_{2}^{2}} s+\alpha_{2} \cos \sqrt{\kappa_{1}^{2}+\kappa_{2}^{2}} s\right)+\frac{\kappa_{2}}{\kappa_{1}} \alpha_{3}
\end{array}\right.
$$

for some constants $\alpha_{1}, \alpha_{2}$ and $\alpha_{3}$. This implies that the helix $\gamma$ is generated by some Killing vector field if and only if $\alpha_{1}=\alpha_{2}=0$. 


\section{Main result}

In this section we give a class of helices with self-intersections in a complex projective plane $\mathbb{C} P^{2}$ with the aid of a well-known isometric parallel imbedding of a 2-dimensional flat torus into $\mathbb{C} P^{2}$ (see [4] for detail). We consider a Riemann surface $N=\left(S^{1} \times S^{1}\right) / \varphi$. Here by representing the first component by $S^{1}=\{z \in \mathbb{C}|| z \mid=1\}$ and the second component by $S^{1}=\left\{\left(a_{1}, a_{2}\right) \in \mathbb{R}^{2} \mid\left(a_{1}\right)^{2}+\left(a_{2}\right)^{2}=1\right\}$, we define the identification $\varphi$ by $\varphi\left(\left(e^{i \theta},\left(a_{1}, a_{2}\right)\right)\right)=\left(-e^{i \theta},\left(-a_{1},-a_{2}\right)\right)$. The Riemannian metric on $N$ is given by $\langle A+\xi, B+\eta\rangle=\frac{2}{9}\langle A, B\rangle_{S^{1}}+\frac{2}{3}\langle\xi, \eta\rangle_{S^{1}}$ for tangent vectors $A, B \in T S^{1}$ of the first component and tangent vectors $\xi, \eta \in T S^{1}$ of the second component, where $\langle,\rangle_{S^{1}}$ denotes the canonical metric of $S^{1}$. We define an isometric imbedding of $N$ into $\mathbb{C} P^{2}(4)$ by

$$
\begin{aligned}
& f\left(e^{i \theta},\left(a_{1}, a_{2}\right)\right) \\
& \quad=\pi\left(\frac{1}{3}\left(e^{-\frac{2 i \theta}{3}}+2 a_{1} e^{\frac{i \theta}{3}}\right), \frac{\sqrt{2}}{3}\left(e^{-\frac{2 i \theta}{3}}-a_{1} e^{\frac{i \theta}{3}}\right), \frac{2}{\sqrt{6}} i a_{2} e^{\frac{i \theta}{3}}\right),
\end{aligned}
$$

where $\pi: S^{5}(1) \longrightarrow \mathbb{C} P^{2}(4)$ is the Hopf fibration. This isometric imbed$\operatorname{ding} f$ is parallel, that is, the second fundamental form $\sigma_{f}$ of $f$ is parallel, and totally real. The second fundamental form $\sigma_{f}$ of this imbedding is in fact expressed as follows for every unit vector $w \in T S^{1}(1)$ of the second component;

$$
\left\{\begin{array}{l}
\sigma_{f}(u, u)=-\frac{1}{\sqrt{2}} J u \\
\sigma_{f}(w, w)=\frac{1}{\sqrt{2}} J u \\
\sigma_{f}(u, w)=\frac{1}{\sqrt{2}} J w
\end{array}\right.
$$

where the tangent vector $u \in T S^{1}(1)$ of the first compornent is the normalized vector of $\partial / \partial \theta$.

We now study images of circles in $N$ under this isometric imbedding. As we see in [1], the imbedding $f$ maps each geodesic on $N$ to a circle of curvature $\frac{1}{\sqrt{2}}$ in $\mathbb{C} P^{2}(4)$. This circle does not have self-intersections, but it is not necessarily closed in $\mathbb{C} P^{2}(4)$. For images of circles on $N$ through $f$ we have the following. 
Proposition 1 For a circle $\gamma$ of curvature $k(>0)$ on $N$, the curve $f \circ \gamma$ is a helix of order 4 in $\mathbb{C} P^{2}(4)$. More precisely,

(1) when $k=\frac{1}{2}$, it is a helix of proper order 3 with curvatures

$$
\kappa_{1}=\frac{\sqrt{3}}{2}, \kappa_{2}=\sqrt{\frac{3}{2}}
$$

(2) when $k \neq \frac{1}{2}$, it is a helix of proper order 4 with curvatures

$$
\kappa_{1}=\sqrt{k^{2}+\frac{1}{2}}, \kappa_{2}=\frac{3 k}{\sqrt{2 k^{2}+1}}, \kappa_{3}=\frac{\left|4 k^{2}-1\right|}{\sqrt{2\left(2 k^{2}+1\right)}} .
$$

Proof. Let $\widetilde{\nabla}$ and $\nabla$ denote the Riemannian connections of $\mathbb{C} P^{2}(4)$ and $N$, respectively. Suppose a circle $\gamma$ of curvature $k$ in $N$ satisfies the following equations:

$$
\nabla_{X} X=k Y \text { and } \nabla_{X} Y=-k X, \text { with } X=V_{1}=\dot{\gamma}
$$

We can represent the orthonormal pair $\{X, Y\}$ as

$$
\left\{\begin{array}{l}
X=\cos \phi \cdot u+\sin \phi \cdot w \\
Y=-\sin \phi \cdot u+\cos \phi \cdot w \quad(0 \leqq \phi<2 \pi),
\end{array}\right.
$$

at each point $\gamma(s)$. We hence find that $\sigma_{f}(X, X)=-\sigma_{f}(Y, Y)$. By the Gauss formula we find that the curve $f \circ \gamma$ satisfies

$$
\tilde{\nabla}_{V_{1}} V_{1}=\kappa_{1} V_{2}, \text { where } \kappa_{1}=\sqrt{k^{2}+\frac{1}{2}} \text { and } V_{2}=\frac{1}{\kappa_{1}}(k Y+\sigma(X, X)) .
$$

Since $f$ has parallel second fundamental form, we get by use of the Gauss formula and (2.3) that

$$
\widetilde{\nabla}_{V_{1}} V_{2}=-\kappa_{1} V_{1}+\kappa_{2} V_{3}
$$

where

$$
\kappa_{2}=\frac{3 k}{\sqrt{2 k^{2}+1}} \quad \text { and } \quad V_{3}=\sqrt{2} \sigma(X, Y)
$$

When $k \neq \frac{1}{2}$, by routine calculations we find with (2.4) that

$$
\tilde{\nabla}_{V_{1}} V_{3}=-\kappa_{2} V_{2}+\kappa_{3} V_{4} \text { and } \tilde{\nabla}_{V_{1}} V_{4}=-\kappa_{3} V_{3}
$$


where

$$
\left\{\begin{aligned}
& \kappa_{3}= \frac{\left|4 k^{2}-1\right|}{\sqrt{2\left(2 k^{2}+1\right)}}, \\
& V_{4}=\frac{\sqrt{2\left(2 k^{2}+1\right)}}{\left|4 k^{2}-1\right|}\left\{\left(\frac{3 \sqrt{2} k^{2}}{2 k^{2}+1}-\frac{1}{\sqrt{2}}\right) Y\right. \\
&\left.\quad+\sqrt{2} k\left(\frac{3}{2 k^{2}+1}-2\right) \sigma(X, X)\right\} .
\end{aligned}\right.
$$

When $k=\frac{1}{2}$, since our calculations go through without the field $V_{4}$, we obtain the conclusion.

Next we investigate properties of these helices. We call a Frenet curve $\rho$ parametrized by its arclength closed, if there exists some $s_{0}(\neq 0)$ satisfying $\rho\left(s+s_{0}\right)=\rho(s)$ for every $s$. We call such the minimum positive $s_{0}$ the length of this closed curve.

Theorem 1 Let $f: N \longrightarrow \mathbb{C} P^{2}(4)$ denote the imbedding defined by (2.1) and $\gamma$ be a circle of curvature $k(>0)$ in $N$. Then we have the following:

(1) The helix $f \circ \gamma$ is closed of length $\frac{2 \pi}{k}$, and is not generated by any Killing vector field on $\mathbb{C} P^{2}(4)$.

(2) The helix $f \circ \gamma$ has self-intersections if and only if $k \leqq \frac{3}{\sqrt{2} \pi}$. The number of intersection points is greater than 2.

Proof. Consider the universal Riemannian covering $p: \mathbb{R}^{2} \longrightarrow N$. Regarding the Riemannian metric on $N$ we can choose a fundamental region for $N$ in $\mathbb{R}^{2}$ as $\mathfrak{F}=\left[0, \frac{2 \sqrt{2}}{3} \pi\right) \times\left[0, \frac{\sqrt{6}}{3} \pi\right)$. Two points $\left(x_{1}, x_{2}\right)$ and $\left(y_{1}, y_{2}\right)$ on $\mathbb{R}^{2}$ satisfies $p\left(\left(x_{1}, x_{2}\right)\right)=p\left(\left(y_{1}, y_{2}\right)\right)$ if and only if either

i) $x_{1}-y_{1}=\frac{2 \sqrt{2}}{3} m_{1} \pi, x_{2}-y_{2}=\frac{2 \sqrt{6}}{3} m_{2} \pi$ for some $m_{1}, m_{2} \in \mathbb{Z}$, or

ii) $x_{1}-y_{1}=\frac{\sqrt{2}}{3}\left(2 m_{1}+1\right) \pi, x_{2}-y_{2}=\frac{\sqrt{6}}{3}\left(2 m_{2}+1\right) \pi$, for some $m_{1}, m_{2} \in \mathbb{Z}$. Let $\tilde{\gamma}$ denote a covering circle of $\gamma$ in $\mathbb{R}^{2}$, which is a circle with radius $\frac{1}{k}$ in the sense of Euclidean Geometry. This guarantees that $\gamma$ is a closed curve of length $\frac{2 \pi}{k}$.

We shall show that $\gamma$ has self-intersections in the case of $k \leq \frac{3}{\sqrt{2} \pi}$. The lift $\widetilde{\gamma}(s)=\left(\widetilde{\gamma_{1}}(s), \widetilde{\gamma_{2}}(s)\right)$ is represented as

$$
\left\{\begin{array}{l}
\widetilde{\gamma_{1}}(s)=\frac{1}{k}\left(v_{2} \cos k s+v_{1} \sin k s\right)-\frac{v_{2}}{k}+\widetilde{\gamma_{1}}(0), \\
\widetilde{\gamma_{2}}(s)=\frac{1}{k}\left(-v_{1} \cos k s+v_{2} \sin k s\right)+\frac{v_{1}}{k}+\widetilde{\gamma_{2}}(0),
\end{array}\right.
$$


where $\left(v_{1}, v_{2}\right) \in \mathbb{R}^{2}$ denotes the unit tangent vector $\dot{\tilde{\gamma}}(0)$. If $\gamma\left(s_{0}\right)=\gamma(0)$ $\left(s_{0} \neq 0\right)$, then $\widetilde{\gamma}\left(s_{0}\right)$ and $\widetilde{\gamma}(0)$ satisfy either the condition i) or ii). When they satisfy the condition i), we have

$$
\left\{\begin{array}{l}
\sin k s_{0}=\frac{2 \sqrt{2}}{3} m_{1} \pi v_{1} k+\frac{2 \sqrt{6}}{3} m_{2} \pi v_{2} k, \\
\cos k s_{0}=\frac{2 \sqrt{2}}{3} m_{1} \pi v_{2} k-\frac{2 \sqrt{6}}{3} m_{2} \pi v_{1} k+1,
\end{array}\right.
$$

hence

$$
\pi k=\frac{3\left(-\sqrt{2} v_{2} m_{1}+\sqrt{6} v_{1} m_{2}\right)}{2\left(m_{1}^{2}+3 m_{2}^{2}\right)}
$$

for some integers $m_{1}, m_{2}$ with $\left(m_{1}, m_{2}\right) \neq(0,0)$. Similarly, when they satisfy the condition ii), we have

$$
\pi k=\frac{3\left\{-\sqrt{2}\left(2 m_{1}+1\right) v_{2}+\sqrt{6}\left(2 m_{2}+1\right) v_{1}\right\}}{\left(2 m_{1}+1\right)^{2}+3\left(2 m_{2}+1\right)^{2}},
$$

for some integers $m_{1}, m_{2}$ with $\left(m_{1}, m_{2}\right) \neq(0,0)$. Conversely, if there exists a pair of integers $\left(m_{1}, m_{2}\right)(\neq(0,0))$ satisfying either $[2.5)$ or $(2.6)$ for some $\left(v_{1}, v_{2}\right) \in \mathbb{R}^{2}$ with $v_{1}^{2}+v_{2}^{2}=1$, we find $\gamma$ has a self-intersection. The number of intersection points corresponds to the cardinality of pairs $\left(v_{1}, v_{2}\right)$ with such properties. Namely, the circle $\gamma$ has self-intersections if and only if some of the images of its covering circles in $\mathbb{R}^{2}$ cut each other in $\mathfrak{F}$. When $k>\frac{3}{\sqrt{2} \pi}$, every covering circle of $\gamma$ is contained in a fundamental domain. Hence $\gamma$ does not have self-intersections. When $k=\frac{3}{\sqrt{2} \pi}$, some two covering circles have a point of contact (see the Figure). This corresponds to the fact that for $\left(v_{1}, v_{2}\right)=\left(\frac{\sqrt{3}}{2},-\frac{1}{2}\right),\left(\frac{\sqrt{3}}{2}, \frac{1}{2}\right),(0,1)$ the equation (2.5) holds with $\left(m_{1}, m_{2}\right)=(0,1),(0,-1),(-1,0)$, respectively. Thus one can easily find that $\gamma$ has self-intersections if and only if $k \leqq \frac{3}{\sqrt{2} \pi}$ and that the number of self-intersection points is greater than 2 in this case. Since $f: N \longrightarrow \mathbb{C} P^{2}(4)$ is an isometric imbedding, we get that $f \circ \gamma$ inherits these properties.

To see that $f \circ \gamma$ is not generated by any Killing vector field on $\mathbb{C} P^{2}(4)$, we compute the complex torsion $\tau_{12}=\left\langle V_{1}, J V_{2}\right\rangle$. As $f$ is totally real, we obtain that $\tau_{12}=\frac{1}{\kappa_{1}}\left\langle X, J \cdot \sigma_{f}(X, X)\right\rangle$. We here make use of the representation (2.4). We set $\phi_{0}$ as the angle between $\dot{\tilde{\gamma}}(0)$ and the positive direction of the first component in $\tilde{N}=\mathbb{R}^{2}$. The angle between $\dot{\tilde{\gamma}}(s)$ and the positive 


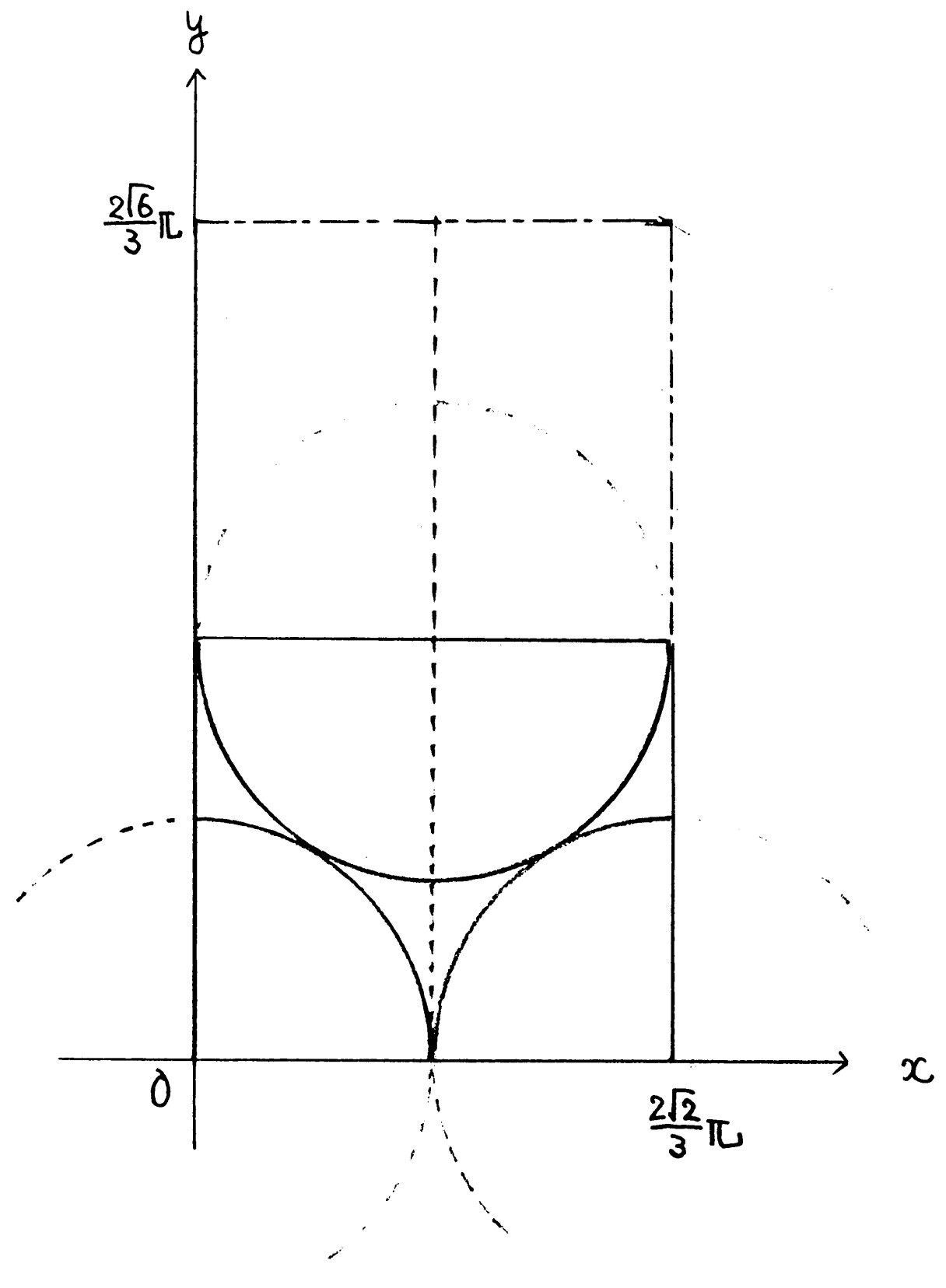

Figure

direction of the first component is then $\phi=k s+\phi_{0}$. We may hence use this angle in (2.4). It follows from (2.2) that

$$
\left\{\begin{array}{l}
\sigma_{f}(X, X)=-\sigma_{f}(Y, Y)=\frac{1}{\sqrt{2}}(-\cos 2 \phi \cdot J u+\sin 2 \phi \cdot J w), \\
\sigma_{f}(X, Y)=\frac{1}{\sqrt{2}}(\sin 2 \phi \cdot J u+\cos 2 \phi \cdot J w)
\end{array}\right.
$$


Thus we obtain that $\tau_{12}=\frac{1}{\sqrt{2 k^{2}+1}} \cos 3 \phi$. Since $\tau_{12}$ is not constant, we find with Fact that $f \circ \gamma$ is not generated by any holomorphic Killing vector field. As Killing vector fields on a complex projective space are holomorphic, we get the conclusion.

Figure shows self-intersections of the closed circle $\gamma$ of curvature $k=$ $\frac{3}{\sqrt{2} \pi}$ in $N$.

For the sake of completeness we here compute all the complex torsions of $f \circ \gamma$. When $k>\frac{1}{2}$, we can see that

$$
\begin{aligned}
\tau_{24}=\frac{1}{\kappa_{1} \kappa_{3}}\left\{\sqrt{2}\left(\frac{3}{2 k^{2}+1}-2\right) k^{2}\right. \\
\\
\left.\quad-\left(\frac{3 \sqrt{2} k^{2}}{2 k^{2}+1}-\frac{1}{\sqrt{2}}\right)\right\}\left\langle Y, J \cdot \sigma_{f}(X, X)\right\rangle,
\end{aligned}
$$

which, together with (2.4) and (2.7), shows that $\tau_{24}=\sin 3\left(k s+\phi_{0}\right)$. When $k<\frac{1}{2}$, we find $\tau_{24}=-\sin 3\left(k s+\phi_{0}\right)$. By the same calculation we obtain the following.

Proposition 2 Let $\gamma$ be a circle of curvature $k(>0)$ in $N$. The complex torsions $\tau_{i j}(s)=\left\langle V_{i}(s), J V_{j}(s)\right\rangle(1 \leq i<j \leq 4)$ of $f \circ \gamma$ is described as follows:

(1) When $k>\frac{1}{2}$, we have

$$
\begin{aligned}
& \tau_{12}=\tau_{34}=\frac{1}{\sqrt{2 k^{2}+1}} \cos 3\left(k s+\phi_{0}\right), \\
& \tau_{13}=-\tau_{24}=-\sin 3\left(k s+\phi_{0}\right), \\
& \tau_{14}=\tau_{23}=-\frac{\sqrt{2} k}{\sqrt{2 k^{2}+1}} \cos 3\left(k s+\phi_{0}\right) .
\end{aligned}
$$

(2) When $k=\frac{1}{2}$, we have

$$
\begin{aligned}
\tau_{12} & =\sqrt{\frac{2}{3}} \cos 3\left(\frac{1}{2} s+\phi_{0}\right), \quad \tau_{13}=-\sin 3\left(\frac{1}{2} s+\phi_{0}\right), \\
\tau_{23} & =-\frac{1}{\sqrt{3}} \cos 3\left(\frac{1}{2} s+\phi_{0}\right) .
\end{aligned}
$$

(3) When $k<\frac{1}{2}$, we have

$$
\tau_{12}=-\tau_{34}=\frac{1}{\sqrt{2 k^{2}+1}} \cos 3\left(k s+\phi_{0}\right),
$$




$$
\begin{aligned}
& \tau_{13}=\tau_{24}=-\sin 3\left(k s+\phi_{0}\right), \\
& \tau_{14}=-\tau_{23}=\frac{\sqrt{2} k}{\sqrt{2 k^{2}+1}} \cos 3\left(k s+\phi_{0}\right) .
\end{aligned}
$$

Here, $\phi_{0}$ is the angle between $\dot{\gamma}(0)$ and the unit vector $u$ tangent to the first component of $N$.

\section{Helices and Veronese imbeddings}

To obtain another class of helices in $\mathbb{C} P^{2}$ we use in this section an isometric parallel holomorphic imbedding of $\mathbb{C} P^{1}\left(\frac{c}{2}\right)\left(=S^{2}\left(\frac{c}{2}\right)\right)$ into $\mathbb{C} P^{2}(c)$. We define $h: S^{2}\left(\frac{c}{2}\right) \longrightarrow \mathbb{C} P^{2}(c)$ by $h\left(z_{0}, z_{1}\right)=\left(z_{0}^{2}, \sqrt{2} z_{0} z_{1}, z_{1}^{2}\right)$, where $z_{0}$, $z_{1}$ are homogeneous coordinates in $\mathbb{C} P^{1}\left(\frac{c}{2}\right)$. The second fundamental form $\sigma_{h}$ of $h$ satisfies

$$
\left\|\sigma_{h}(X, X)\right\|=\frac{\sqrt{c}}{2}
$$

for every unit vector $X$ (see [5]). This imbedding $h$ maps each geodesic on $S^{2}\left(\frac{c}{2}\right)$ to a circle of curvature $\frac{\sqrt{c}}{2}$ in $\mathbb{C} P^{2}(c)$. This circle is simple and its length is $\frac{2 \sqrt{2}}{\sqrt{c}} \pi$. It lies on $\mathbb{R} P^{2}\left(\frac{c}{4}\right)$ which is a totally real totally geodesic submanifold of $\mathbb{C} P^{2}(c)$ (see, [1]).

We here study geometric properties of images of circles in $\mathbb{C} P^{1}\left(\frac{c}{2}\right)$ under the isometric imbedding $h$.

Proposition 3 For a circle $\gamma$ of curvature $k(>0)$ in $\mathbb{C} P^{1}\left(\frac{c}{2}\right)$, the curve $h \circ \gamma$ is a helix of order 4 in $\mathbb{C} P^{2}(c)$. More precisely

(1) when $k=\frac{\sqrt{c}}{2 \sqrt{2}}$, it is a helix of proper order 3 with curvatures $\kappa_{1}=\frac{\sqrt{3 c}}{2 \sqrt{2}}$,

$$
\kappa_{2}=\frac{\sqrt{3 c}}{2} \text {, and }
$$

(2) when $k \neq \frac{\sqrt{c}}{2 \sqrt{2}}$ it is a helix of proper order 4 with curvatures

$$
\kappa_{1}=\sqrt{k^{2}+\frac{c}{4}}, \kappa_{2}=\frac{3 k \sqrt{c}}{\sqrt{4 k^{2}+c}}, \kappa_{3}=\frac{\left|8 k^{2}-c\right|}{2 \sqrt{4 k^{2}+c}} .
$$

Proof. We use the same terminologies as in the proof of Proposition 1. By the Gauss formula and (3.1) the curve $h \circ \gamma$ satisfies

$$
\tilde{\nabla}_{V_{1}} V_{1}=\kappa_{1} V_{2}, \text { where } \kappa_{1}=\sqrt{k^{2}+\frac{c}{4}} \text { and } V_{2}=\frac{1}{\kappa_{1}}\left(k Y+\sigma_{h}(X, X)\right) \text {. }
$$

As $Y= \pm J X$ and $h$ is holomorphic, we have $\sigma_{h}(X, Y)= \pm J \sigma_{h}(X, X)$. Since $h$ has parallel second fundamental form, we get by direct calculations 
that

$$
\tilde{\nabla}_{V_{1}} V_{2}=-\kappa_{1} V_{1}+\kappa_{2} V_{3}, \quad \text { with } \kappa_{2}=\frac{3 k \sqrt{c}}{\sqrt{4 k^{2}+c}}, \quad V_{3}=\frac{2}{\sqrt{c}} \sigma_{h}(X, Y) .
$$

Continuing routine calculations we obtain the following when $k \neq \frac{\sqrt{c}}{2 \sqrt{2}}$ :

$$
\left\{\begin{array}{l}
\tilde{\nabla}_{V_{1}} V_{3}=-\kappa_{2} V_{2}+\kappa_{3} V_{4} \\
\tilde{\nabla}_{V_{1}} V_{4}=-\kappa_{3} V_{3}
\end{array}\right.
$$

with

$$
\kappa_{3}=\frac{\left|8 k^{2}-c\right|}{2 \sqrt{4 k^{2}+c}}, \quad V_{4}=\frac{4}{\sqrt{c\left(4 k^{2}+c\right)}}\left(\frac{c}{4} Y-k \cdot \sigma_{h}(X, X)\right) .
$$

When $k=\frac{\sqrt{c}}{2 \sqrt{2}}$, we find $\tilde{\nabla}_{V_{1}} V_{3}=-\kappa_{2} V_{2}$, so that we get the conclusion.

For properties of these helices we have the following.

Theorem 2 For a circle $\gamma$ of curvature $k$ in $\mathbb{C} P^{1}\left(\frac{c}{2}\right)$, the helix $h \circ \gamma$ is simple and closed with length $\frac{2 \pi}{\sqrt{k^{2}+\frac{c}{2}}}$, and is generated by a Killing vector field in $\mathbb{C} P^{2}(c)$. The complex torsions $\tau_{i j}(s)=\left\langle V_{i}(s), J V_{j}(s)\right\rangle(1 \leq i<j \leq$ 4) of $h \circ \gamma$ are described as follows according as the complex torsion of $\gamma$ is \pm 1 (i.e. $\left.\nabla_{\dot{\gamma}} \dot{\gamma}=\mp k J \dot{\gamma}\right)$ :

(1) When $k>\frac{\sqrt{c}}{2 \sqrt{2}}$, we have $\tau_{12}=\tau_{34}=\frac{ \pm k}{\sqrt{k^{2}+\frac{c}{4}}}, \tau_{23}=\tau_{14}=\frac{ \pm \sqrt{c}}{\sqrt{4 k^{2}+c}}$, $\tau_{13}=\tau_{24}=0$.

(2) When $k=\frac{\sqrt{c}}{2 \sqrt{2}}$, we have $\tau_{12}= \pm \frac{1}{\sqrt{3}}, \tau_{23}= \pm \frac{\sqrt{2}}{\sqrt{3}}, \tau_{13}=0$.

(3) When $k<\frac{\sqrt{c}}{2 \sqrt{2}}$, we have $\tau_{12}=-\tau_{34}=\frac{ \pm k}{\sqrt{k^{2}+\frac{c}{4}}}, \tau_{23}=-\tau_{14}=\frac{ \pm \sqrt{c}}{\sqrt{4 k^{2}+c}}$, $\tau_{13}=\tau_{24}=0$.

Proof. It is known that every circle of curvature $k(\geq 0)$ on an $n$-dimensional sphere $S^{n}(c)$ of curvature $c$ is a simple closed curve with length $\frac{2 \pi}{\sqrt{k^{2}+c}}$. Since $h$ is an isometric imbedding, we get that $h \circ \gamma$ is simple and closed with length $\frac{2 \pi}{\sqrt{k^{2}+\frac{c}{2}}}$.

As the imbedding $h$ is holomorphic we have

$$
\tau_{13}=\left\langle V_{1}, J V_{3}\right\rangle=\frac{2}{\sqrt{c}}\left\langle X, J \cdot \sigma_{h}(X, Y)\right\rangle=0 .
$$


By routine calculations we get the desired expression for other $\tau_{i j}$. Since all its complex torsions are constant, we find by Fact that it is generated by some Killing vector field in $\mathbb{C} P^{2}(c)$.

The authors determined in [2] helices of proper order 4 which are generated by Killing vector fields in $\mathbb{C} P^{2}(c)$ : For given arbitrary positive constants $k_{1}, k_{2}$ and $k_{3}$, there exist four equivalence classes of helices which are generated by Killing vector fields of proper order 4 with curvatures $k_{1}, k_{2}$ and $k_{3}$ with respect to holomorphic isometries of $\mathbb{C} P^{2}(c)$. The complex torsions $\tau_{i j}(i \leq i<j \leq 4)$ of these four classes of helices satisfy one of the following (i), (ii) or one of the following (i), (ii'):

(i) $\tau_{12}=\tau_{34}=\tau, \tau_{23}=\tau_{14}=\frac{k_{2} \tau}{k_{1}+k_{3}}, \tau_{13}=\tau_{24}=0$, where $\tau=$ $\pm \frac{k_{1}+k_{3}}{\sqrt{k_{2}^{2}+\left(k_{1}+k_{3}\right)^{2}}}$.

(ii) $\tau_{12}=-\tau_{34}=\tau, \tau_{23}=-\tau_{14}=\frac{k_{2} \tau}{k_{1}-k_{3}}, \tau_{13}=\tau_{24}=0$, when $k_{1} \neq k_{3}$, where $\tau= \pm \frac{k_{1}-k_{3}}{\sqrt{k_{2}^{2}+\left(k_{1}-k_{3}\right)^{2}}}$.

(ii') $\tau_{12}=\tau_{34}=\tau_{13}=\tau_{24}=0, \tau_{23}=-\tau_{14}= \pm 1$, when $k_{1}=k_{3}$.

We finally point out the following.

(1) When $k>\frac{\sqrt{c}}{2 \sqrt{2}}$, the complex torsions of the helix $h \circ \gamma$ in Theorem 2 satisfy the case (i).

(2) When $k<\frac{\sqrt{c}}{2 \sqrt{2}}$, the complex torsions of the helix $h \circ \gamma$ in Theorem 2 satisfy the case (ii).

\section{References}

[1] Adachi T., Maeda S. and Udagawa S., Circles in a complex projective space. Osaka J. Math. 32 (1995), 1197-1202.

[2] Adachi T. and Maeda S., Holomorphic helices in a complex space form. Proc. Amer. Math. Soc. 125 (1997), 1197-1202.

[3] Maeda S. and Ohnita Y., Helical geodesic immersions into complex space forms. Geometriae Dedicata 30 (1989), 93-114.

[4] Naitoh H., Isotropic submanifolds with parallel second fundamental form in $P^{m}(c)$. Osaka J. Math. 18 (1981), 427-464.

[5] Ogiue K., Differential geometry of Kaehler submanifolds. Advances in Mathematics 13 (1974), 73-114. 
Toshiaki Adachi

Department of Mathematics

Nagoya institute of Technology

Nagoya 466-8555, Japan

E-mail: adachi@math.kyy.nitech.ac.jp

Sadahiro Maeda

Department of Mathematics

Shimane University

Matsue 690-8504, Japan

E-mail: smaeda@math.shimane-u.ac.jp 